\title{
Effect of Variable Levels of Metabolisable Energy and Metabolisable Protein On Growth, Immunity and Health Performance in Male Murrah Buffalo Calves (Bubalus Bubalis)
}

Prince Chauhan ( $\sim$ prince35chauhan@gmail.com )

National Dairy Research Institute https://orcid.org/0000-0003-1493-099X

Raman Malik

National Dairy Research Institute

Shambhvi Shambhvi

National Dairy Research Institute

Dipak Dey

National Dairy Research Institute

Zahid Bashir

National Dairy Research Institute

\section{Research Article}

Keywords: Buffalo, Metabolisable Energy, Metabolisable protein, Growth, Supplementation, Immunity, Health.

Posted Date: October 25th, 2021

DOI: https://doi.org/10.21203/rs.3.rs-752038/v1

License: (c) (i) This work is licensed under a Creative Commons Attribution 4.0 International License.

Read Full License 


\section{Abstract}

In calve nutrition; malnutrition is still the one of the limiting factor in developing countries (Olafadehan and Adewumi 2009). Poor nutrition and imbalance nutrition supply primarily effects health and production of the animal which rendered the animal to reach its maximum genetic potential level. To understand the effects of different level of dietary energy and protein on health and immunity of the ruminant, the present study was considered to investigate the effects of different levels of ME and MP on body weight changes, dry matter intake, feed conversation efficiency and various immunity and health parameters. For growth trial, Twenty four calves were divided into four groups with six animals $(n=6)$ in each group. All animals were fed individually and for feeding four types of concentrates were prepared; Treatment 1 (control, in accordance with ICAR 2013 feeding standard), Treatment 2 (T2, 30\% extra metabolisable energy above ICAR 2013 feeding standard), Treatment 3 (30\% extra metabolisable protein above ICAR 2013 feeding standard) and T4 (30\% extra metabolisable protein and 30\% extra metabolisable energy). The growth trial was conducted for 12 months and during this trial period dry matter intake was measured daily, body weight measured fortnightly and blood was collected on monthly interval for evaluating blood immunity parameters.

There was no significant alteration in average body weight changes when compared between groups. The overall mean of total DMI $(\mathrm{kg})$ for T1, T2, T3 and T4 groups were $6.86 \pm 0.49,6.49 \pm 0.49,6.66 \pm 0.51$ and $6.68 \pm 0.48$ respectively. There was no significant variation in GPx, SOD, catalase, cortisol and IgG due to different levels of ME and MP. The study outcomes states providing higher energy and protein did not caused any stress and any alteration in various health parameters in Murrah buffalo calves during growth period.

\section{Introduction}

The buffaloes are considered as the main dairy animals in India, contributing $51 \%$ to the total milk production of the country (BAHFS, 2015). Diversity of the buffaloes in India is represented by 13 registered and well defined breeds' along with non-descript populations; Murrah is the prominent buffalo breed in the country followed by Surti, Mehesana and Jafarabadi breeds (Vohra et. al., 2016). In tropical countries like India, buffaloes showed better efficiency in utilization of nutrients over cattle and had comparatively higher resistance to disease (Paul et. al., 2003). In calve nutrition; malnutrition is still the one of the limiting in developing countries (Olafadehan and Adewumi 2009). Apart from genetics, undernutrition and imbalance feeding are the major causes of lower growth rate (Bhatti et. al., 2007).

As breeding bull sires the whole herd so it is considered as half of the herd but generally bull's nutrition is neglected. The nutrition of the buffalo bulls has important influence on the quantity and quality of the semen. Under-nutrition which includes mainly deficiency of major and minor nutrients is the most common cause of poor growth rate. 
One of the major dietary nutrients which influence the efficiency of other nutrients in feed is dietary energy which also effects growth and production by the animal (Hosseini et. al., 2008). The other most important dietary nutrient effecting reproductive performances of bulls is the available digestible protein in the diet. The feed with deficiency in protein and energy causes rapid weight loss which in turn influences the productive life of bull (Mecham et. al.,1963). Therefore, the optimum energy and protein levels for better growth and better health of young buffalo male needs to be critically understood, so that male bull productive life can be increased.

Keeping in view, the benefits of feeding higher energy and protein on growth, production and immunity from early age and during growth trial and this study was undertaken to determine the effects of feed energy and protein levels on body weight changes, total dry matter intake, health and immunity performance of growing Murrah buffalo calves.

\section{Material And Methods}

The study was carried out at Livestock Research Centre of National Dairy Research Institute (N.D.R.I.), Karnal, Haryana, India. Twenty four male Murrah calves were selected and distributed randomly into four groups ( 6 animals each) according to their body weight so that average body weight of each group was similar at the time of starting of experiment. Four types of concentrates were prepared; Treatment 1 (control, in accordance with ICAR 2013 feeding standard), Treatment 2 (T2, 30\% extra metabolisable energy above ICAR 2013 feeding standard), Treatment 3 (30\% extra metabolisable protein above ICAR 2013 feeding standard) and T4 (30\% extra metabolisable protein and 30\% extra metabolisable energy). Concentrate mixture, green fodder (sugargraze) and dry roughage (wheat straw) were supplied at the ratio of 40: 40: 20 (on dry matter basis) in all three types of TMRs. Blood samples were collected at monthly interval during experiment period to elicit the effect of higher energy and protein level.

DM intake was recorded daily by subtracting the residual DM from the quantity of DM offered. To estimate body weight, the animals were weighed before feeding and watering in the morning on two consecutive days at the start of experimental feeding and thereafter at fortnightly intervals during the entire experimental period. Blood samples $(8-10 \mathrm{ml})$ were collected in sterile heparinised vacutainer tubes (vacuette, Greiner bio-one, $\mathrm{GmbH}$, Austria) from jugular vein puncture, causing least stress to the animal. Blood samples were taken on monthly intervals. Immediately after collection, tubes were kept in ice and transported to the laboratory for further processing.

\section{Assessment of antioxidant status}

Antioxidant enzymes (superoxide dismutase (SOD), catalase and glutathione peroxidase $\left(\mathrm{GP}_{\mathrm{X}}\right)$ ) were estimated using RBC hemolysate within three days after collection of blood.

\subsubsection{Preparation of blood lysate}


Erythrocytes and erythrocyte membranes are more vulnerable to lipid peroxidation due to constant exposure to high oxygen tension and richness in polyunsaturated fatty acid, respectively. Besides this, the erythrocytes contain several defense mechanisms against oxidative stress which include both enzymatic and non-enzymatic antioxidants. The simplicity, availability and ease of isolation make erythrocytes as an excellent model for in vivo studies on cellular antioxidant response to oxidative stress under clinical conditions and preparation of hemolysate is an initial step in the analysis of different oxidative stress markers.

\section{Principle}

Red blood cells when placed in a hypotonic (with a lower solute concentration than that is found in the cells) solution, it rapidly moves into the cells by osmosis, causing the cells to swell and burst releasing hemoglobin in the surroundings. This phenomenon is known as hemolysis. This fresh haemolysate was further used for estimation of various enzymatic and non-enzymatic antioxidants.

\section{Reagents}

Acid citrate dextrose (ACD): for $250 \mathrm{~mL}$ in doubled distilled water (DDW)

Citric acid: $2.0 \mathrm{~g}$

Sodium citrate: $5.5 \mathrm{~g}$

Dextrose: $6.125 \mathrm{~g}$

The volume was made to $250 \mathrm{~mL}$ with DDW

EDTA stabilising solution: for $500 \mathrm{~mL}$ in DDW

$\operatorname{EDTA}(2.7 \mathrm{mM}, \mathrm{pH}-7.0): 0.5025 \mathrm{~g}$

Beta-mercaptoethanol (0.7mM): $24.55 \mu \mathrm{L}$

Dissolved EDTA and set $\mathrm{pH}$ at 7.0 then added beta-mercaptoethanol and finally made up volume to 500 $\mathrm{mL}$.

Normal saline (0.9\%): for this $4.5 \mathrm{~g} \mathrm{NaCl}$ (sodium chloride) was dissolved in DDW to make the volume up to $500 \mathrm{~mL}$.

\section{Procedure}

Blood was collected in ACD (at $1.5 \mathrm{~mL} / 10 \mathrm{~mL}$ of blood) in $2.0 \mathrm{~mL}$ eppendrof tube.

Sample was thoroughly mixed with the anticoagulant ACD.

Samples were centrifuged in refrigerated conditions $\left(4-6^{\circ} \mathrm{C}\right)$ at $2500 \mathrm{rpm}$ for $20 \mathrm{~mm}$. 
Supernatant (plasma) and buffy coat was carefully removed.

$1 \mathrm{~mL}$ of ice-cold normal saline was added to packed RBC in each tube which was gently mixed by up and down movements 2-4 times.

Samples were again centrifuged at $4-6^{\circ} \mathrm{C}$ for $20 \mathrm{~min}$. at $2500 \mathrm{rpm}$.

Above steps were repeated thrice.

$0.5 \mathrm{~mL}$ of packed erythrocytes were taken and an equal quantity of normal saline was added to form RBC suspension.

$0.2 \mathrm{~mL}$ of RBC suspension was taken in a separate $2 \mathrm{~mL}$ vial and $1.8 \mathrm{~mL}$ of ice-cold EDTA stabilizing solution was added to make haemolysate.

If further dilution was required then EDTA stabilising solution was used.

Haemolysate was analysed within three days.

\section{Statistical Analysis}

Analysis of data was done by SPSS (22.0) software using ANOVA (turkey-test). Mean and standard error were calculated and comparisons between and within groups were made.

\section{Results}

\section{Body weight changes}

The data pertaining to change in body weight was measured fortnightly and the values are presented in table 1.The initial body weight $(\mathrm{kg})$ was similar in all four groups. The initial mean body weight $(\mathrm{kg})$ for T1, T2, T3 and T4 groups were 159.82 $\pm 14.62,158.72 \pm 14.10,158.34 \pm 16.70$ and $159.14 \pm 13.32$ respectively. All the groups reached to the mark of $200 \mathrm{~kg}$ mean body weight in 4 months. About eight fortnight mean body weight of T1, T2, T3 and T4 group were 207.09 $\pm 13.37,212.24 \pm 10.99,208.83 \pm$ 15.26 and $218.88 \pm 13.89$ whereas T1 and T3 group reached mean body weight of $200 \mathrm{Kg}$ in eighth fortnight where T2 and T4 group which were supplemented with higher energy reached $200 \mathrm{~kg}$ body weight in seven fortnight. The final body weight $(\mathrm{kg})$ at $25^{\text {th }}$ fortnight for T1, T2, T3 and T4 group were $362.18 \pm 8.74,378.68 \pm 7.96,382.58 \pm 10.63,395.01 \pm 10.09$ respectively and the corresponding value for overall mean value of body weight were $249.22 \pm 11.91,257.67 \pm 9.97,255.91 \pm 13.63,269.30 \pm 13.52$ respectively. Body weight at last fortnight and overall mean of body weight indicates numerically higher body weight achieved in T4 group however change is not significantly higher. The mean body weight did not varied significantly in any of the fortnight or in the overall mean value.

Table 1. Effect of different levels of dietary ME and MP on body weight changes (kg) fortnightly 


\begin{tabular}{|c|c|c|c|c|c|c|}
\hline Fortnight & $\mathrm{T} 1$ & T2 & T3 & $\mathrm{T} 4$ & SEM & P-value \\
\hline 1 & $\begin{array}{l}159.82 \pm \\
14.62\end{array}$ & $158.72 \pm 14.10$ & $158.34 \pm 16.70$ & $159.14 \pm 13.32$ & 6.87 & 1.000 \\
\hline 2 & $\begin{array}{l}166.47 \pm \\
14.56\end{array}$ & $164.76 \pm 13.96$ & $164.96 \pm 16.47$ & $167.01 \pm 13.42$ & 6.83 & 0.999 \\
\hline 3 & $\begin{array}{l}173.08 \pm \\
13.91\end{array}$ & $171.15 \pm 13.32$ & $172.31 \pm 16.06$ & $175.28 \pm 13.44$ & 6.64 & 0.997 \\
\hline 4 & $\begin{array}{l}178.93 \pm \\
13.70\end{array}$ & $180.06 \pm 12.20$ & $178.98 \pm 15.85$ & $183.23 \pm 13.56$ & 6.49 & 0.996 \\
\hline 5 & $\begin{array}{l}184.78 \pm \\
13.34\end{array}$ & $187.67 \pm 11.95$ & $185.98 \pm 15.61$ & $190.35 \pm 13.87$ & 6.43 & 0.992 \\
\hline 6 & $\begin{array}{l}190.82 \pm \\
13.22\end{array}$ & $194.85 \pm 11.92$ & $191.98 \pm 15.66$ & $198.63 \pm 14.27$ & 6.48 & 0.979 \\
\hline 7 & $\begin{array}{l}198.08 \pm \\
13.42\end{array}$ & $203.58 \pm 11.35$ & $199.62 \pm 15.90$ & $208.22 \pm 14.00$ & 6.47 & 0.954 \\
\hline 8 & $\begin{array}{l}207.09 \pm \\
13.37\end{array}$ & $212.24 \pm 10.99$ & $208.83 \pm 15.26$ & $218.88 \pm 13.89$ & 6.35 & 0.929 \\
\hline 9 & $\begin{array}{l}212.08 \pm \\
13.12\end{array}$ & $219.48 \pm 10.81$ & $213.91 \pm 15.09$ & $227.37 \pm 14.74$ & 6.44 & 0.855 \\
\hline 10 & $\begin{array}{l}217.55 \pm \\
12.94\end{array}$ & $226.35 \pm 9.73$ & $219.63 \pm 14.95$ & $236.87 \pm 15.31$ & 6.45 & 0.741 \\
\hline 11 & $\begin{array}{l}222.40 \pm \\
12.79\end{array}$ & $231.70 \pm 9.55$ & $225.18 \pm 15.02$ & $245.32 \pm 16.17$ & 6.61 & 0.646 \\
\hline 12 & $\begin{array}{l}228.93 \pm \\
12.43\end{array}$ & $238.57 \pm 9.49$ & $231.83 \pm 14.93$ & $254.77 \pm 16.69$ & 6.70 & 0.554 \\
\hline 13 & $\begin{array}{l}237.82 \pm \\
12.38\end{array}$ & $248.78 \pm 9.62$ & $241.60 \pm 13.93$ & $264.40 \pm 16.71$ & 6.60 & 0.525 \\
\hline 14 & $\begin{array}{l}246.92 \pm \\
11.45\end{array}$ & $258.38 \pm 9.64$ & $251.68 \pm 13.18$ & $273.18 \pm 16.33$ & 6.35 & 0.515 \\
\hline 15 & $\begin{array}{l}256.77 \pm \\
11.19\end{array}$ & $269.77 \pm 9.40$ & $262.63 \pm 13.31$ & $284.12 \pm 16.21$ & 6.33 & 0.482 \\
\hline 16 & $\begin{array}{l}267.58 \pm \\
11.45\end{array}$ & $279.95 \pm 8.63$ & $275.13 \pm 13.27$ & $295.45 \pm 15.45$ & 6.18 & 0.462 \\
\hline 17 & $\begin{array}{l}277.98 \pm \\
11.21\end{array}$ & $289.90 \pm 9.67$ & $287.23 \pm 13.23$ & $305.49 \pm 14.31$ & 6.07 & 0.472 \\
\hline 18 & $\begin{array}{l}288.88 \pm \\
10.51\end{array}$ & $298.87 \pm 9.42$ & $299.63 \pm 12.96$ & $316.17 \pm 13.72$ & 5.86 & 0.449 \\
\hline 19 & $\begin{array}{l}299.65 \pm \\
10.05\end{array}$ & $310.55 \pm 9.23$ & $311.12 \pm 11.73$ & $327.69 \pm 13.45$ & 5.64 & 0.388 \\
\hline
\end{tabular}




\begin{tabular}{|lllllll|}
\hline 20 & $\begin{array}{l}309.97 \pm \\
10.60\end{array}$ & $322.95 \pm 8.12$ & $323.52 \pm 11.54$ & $340.06 \pm 13.01$ & 5.58 & 0.313 \\
\hline 21 & $\begin{array}{l}320.92 \pm \\
10.32\end{array}$ & $332.35 \pm 8.59$ & $334.78 \pm 10.92$ & $350.16 \pm 12.55$ & 5.44 & 0.313 \\
\hline 22 & $331.68 \pm 9.99$ & $343.52 \pm 8.27$ & $347.00 \pm 10.38$ & $361.12 \pm 11.63$ & 5.21 & 0.264 \\
\hline 23 & $\begin{array}{l}339.87 \pm \\
10.17\end{array}$ & $353.17 \pm 7.72$ & $359.45 \pm 9.98$ & $371.95 \pm 11.01$ & 5.17 & 0.170 \\
\hline 24 & $350.23 \pm 9.15$ & $365.75 \pm 8.03$ & $369.85 \pm 10.30$ & $382.75 \pm 10.48$ & 5.06 & 0.150 \\
\hline 25 & $362.18 \pm 8.74$ & $378.68 \pm 7.96$ & $382.58 \pm 10.63$ & $395.01 \pm 10.09$ & 5.03 & 0.136 \\
\hline Mean & $\mathbf{2 4 9 . 2 2 \pm}$ & $\mathbf{2 5 7 . 6 7 \pm 9 . 9 7}$ & $\mathbf{2 5 5 . 9 1 \pm 1 3 . 6 3}$ & $\mathbf{2 6 9 . 3 0 \pm 1 3 . 5 2}$ & $\mathbf{5 . 9 5}$ & $\mathbf{0 . 7 1 5}$ \\
\hline
\end{tabular}

The values are Mean \pm SE of observations on five animals in each group

\section{Dry matter intake}

During this study, four ( $T 1, T 2, T 3$ and $T 4)$ different types of concentrates were used to make TMR which was fed to four (T1, T2, T3 and T4) groups and their intakes in different animals were estimated on dry matter basis fortnightly. The result was presented in table 2. At $1^{\text {st }}$ fortnight $\mathrm{DMI}(\mathrm{kg} / \mathrm{d})$ were $5.37 \pm 0.58$, $5.39 \pm 0.56,5.14 \pm 0.62$ and $5.27 \pm 0.48$ for $\mathrm{T} 1, \mathrm{~T} 2, \mathrm{~T} 3$ and $\mathrm{T} 4$ respectively and at $25^{\text {th }}$ (last) fortnight corresponding values were $9.46 \pm 0.33,8.91 \pm 0.35,9.49 \pm 0.43$ and $9.01 \pm 0.44$. The DMl intake was gradually increased throughout the trial which is normal as body weight and requirement increased with progress in age and trial. There were no significant changes among the different group in any of the fortnight as far as total DMI was concern. The overall mean DMI $(\mathrm{kg} / \mathrm{d})$ for $\mathrm{T} 1, \mathrm{~T} 2$, T3 and T4 group were $6.86 \pm 0.49,6.49 \pm 0.49,6.66 \pm 0.51$ and $6.68 \pm 0.48$ respectively which did not showed any significant alteration in between the different groups.

Table 2. Effect of different levels of dietary ME and MP on DM intake (kg/d) fortnightly 


\begin{tabular}{|c|c|c|c|c|c|c|}
\hline Fortnight & $\mathrm{T} 1$ & T2 & T3 & $\mathrm{T} 4$ & SEM & P-value \\
\hline 1 & $5.37 \pm 0.58$ & $5.39 \pm 0.56$ & $5.14 \pm 0.62$ & $5.27 \pm 0.48$ & 0.26 & 0.998 \\
\hline 2 & $5.82 \pm 0.64$ & $5.55 \pm 0.55$ & $5.31 \pm 0.65$ & $5.83 \pm 0.65$ & 0.29 & 0.921 \\
\hline 3 & $5.83 \pm 0.52$ & $5.28 \pm 0.52$ & $5.82 \pm 0.59$ & $5.68 \pm 0.45$ & 0.25 & 0.861 \\
\hline 4 & $6.11 \pm 0.54$ & $5.70 \pm 0.48$ & $5.88 \pm 0.55$ & $5.75 \pm 0.49$ & 0.24 & 0.945 \\
\hline 5 & $5.99 \pm 0.56$ & $5.82 \pm 0.58$ & $5.99 \pm 0.65$ & $5.92 \pm 0.52$ & 0.27 & 0.996 \\
\hline 6 & $6.12 \pm 0.46$ & $5.87 \pm 0.53$ & $5.93 \pm 0.64$ & $5.90 \pm 0.64$ & 0.27 & 0.989 \\
\hline 7 & $6.28 \pm 0.52$ & $5.94 \pm 0.47$ & $5.87 \pm 0.59$ & $5.86 \pm 0.47$ & 0.24 & 0.930 \\
\hline 8 & $6.48 \pm 0.63$ & $6.19 \pm 0.40$ & $6.38 \pm 0.60$ & $6.30 \pm 0.56$ & 0.26 & 0.986 \\
\hline 9 & $5.89 \pm 0.63$ & $5.90 \pm 0.52$ & $5.73 \pm 0.69$ & $6.00 \pm 0.66$ & 0.29 & 0.993 \\
\hline 10 & $6.04 \pm 0.61$ & $5.79 \pm 0.63$ & $5.91 \pm 0.60$ & $5.82 \pm 0.56$ & 0.28 & 0.991 \\
\hline 11 & $6.45 \pm 0.43$ & $5.99 \pm 0.50$ & $5.53 \pm 0.54$ & $6.30 \pm 0.37$ & 0.23 & 0.528 \\
\hline 12 & $5.86 \pm 0.44$ & $5.45 \pm 0.29$ & $6.04 \pm 0.50$ & $5.76 \pm 0.42$ & 0.20 & 0.791 \\
\hline 13 & $6.32 \pm 0.58$ & $6.06 \pm 0.54$ & $6.22 \pm 0.61$ & $6.91 \pm 0.59$ & 0.28 & 0.751 \\
\hline 14 & $7.27 \pm 0.44$ & $6.93 \pm 0.42$ & $6.23 \pm 0.40$ & $6.68 \pm 0.46$ & 0.22 & 0.398 \\
\hline 15 & $6.25 \pm 0.46$ & $6.40 \pm 0.39$ & $6.21 \pm 0.43$ & $6.14 \pm 0.46$ & 0.20 & 0.978 \\
\hline 16 & $6.98 \pm 0.47$ & $6.21 \pm 0.30$ & $6.35 \pm 0.42$ & $6.52 \pm 0.49$ & 0.21 & 0.617 \\
\hline 17 & $7.85 \pm 0.59$ & $7.44 \pm 0.42$ & $7.96 \pm 0.52$ & $7.72 \pm 0.52$ & 0.24 & 0.906 \\
\hline 18 & $7.18 \pm 0.45$ & $6.80 \pm 0.37$ & $7.02 \pm 0.46$ & $6.96 \pm 0.39$ & 0.20 & 0.931 \\
\hline 19 & $7.87 \pm 0.36$ & $7.00 \pm 0.38$ & $7.57 \pm 0.38$ & $7.57 \pm 0.48$ & 0.20 & 0.502 \\
\hline 20 & $8.16 \pm 0.39$ & $8.36 \pm 0.28$ & $8.44 \pm 0.40$ & $8.69 \pm 0.53$ & 0.19 & 0.831 \\
\hline 21 & $7.89 \pm 0.43$ & $6.86 \pm 0.48$ & $7.74 \pm 0.40$ & $7.15 \pm 0.58$ & 0.24 & 0.399 \\
\hline 22 & $8.06 \pm 0.49$ & $7.34 \pm 0.54$ & $7.95 \pm 0.53$ & $7.55 \pm 0.56$ & 0.25 & 0.744 \\
\hline 23 & $7.99 \pm 0.53$ & $7.45 \pm 0.34$ & $7.63 \pm 0.30$ & $7.94 \pm 0.33$ & 0.19 & 0.716 \\
\hline 24 & $8.04 \pm 0.34$ & $7.65 \pm 0.36$ & $8.05 \pm 0.42$ & $7.77 \pm 0.38$ & 0.18 & 0.832 \\
\hline 25 & $9.46 \pm 0.33$ & $8.91 \pm 0.35$ & $9.49 \pm 0.43$ & $9.01 \pm 0.44$ & 0.19 & 0.627 \\
\hline Mean & $6.86 \pm 0.49$ & $6.49 \pm 0.49$ & $6.66 \pm 0.51$ & $6.68 \pm 0.48$ & 0.22 & 0.958 \\
\hline
\end{tabular}

The values are Mean \pm SE of observations on five animals in each group 


\section{Feed conversion ratio}

FCR was calculated for every fortnight and the results are presented in table 3 . At first fortnight FCR for $\mathrm{T} 1, \mathrm{~T} 2, \mathrm{~T} 3$ and T4 were $12.80 \pm 2.11,13.87 \pm 1.95,12.63 \pm 2.33$ and $10.86 \pm 1.74$ respectively. The FCR showed no regular pattern and varied widely between fortnights, whereas in initial period FCR is found to be high in each group indicating adaptation to new feed resulting into poor FCR. At $24^{\text {th }}$ fortnight FCR values for T1, T2, T3 and T4 were $10.28 \pm 0.81,9.07 \pm 0.83,9.60 \pm 0.57$ and $9.86 \pm 1.02$ respectively. FCR value did not varied significantly in any of the fortnight. The overall mean value of FCR for T1 (13.64 \pm 1.51), T2 (11.77 \pm 1.32$)$, T3 (11.99 \pm 1.45$)$ and T4 (11.33 \pm 1.30$)$ did not altered significantly among different group indicates no significant changes in FCR due to higher MP and ME.

Table 3. Effect of different level of dietary ME and MP on FCR fortnightly 


\begin{tabular}{|c|c|c|c|c|c|c|}
\hline Fortnight & $\mathrm{T} 1$ & T2 & T3 & $\mathrm{T} 4$ & SEM & P-value \\
\hline 1 & $12.80 \pm 2.11$ & $13.87 \pm 1.95$ & $12.63 \pm 2.33$ & $10.86 \pm 1.74$ & 0.98 & 0.774 \\
\hline 2 & $15.63 \pm 3.92$ & $14.54 \pm 2.93$ & $12.02 \pm 2.38$ & $10.97 \pm 1.46$ & 1.37 & 0.629 \\
\hline 3 & $16.13 \pm 2.85$ & $10.73 \pm 2.29$ & $14.25 \pm 2.63$ & $11.24 \pm 1.64$ & 1.21 & 0.359 \\
\hline 4 & $16.74 \pm 2.33$ & $12.28 \pm 2.11$ & $13.66 \pm 2.36$ & $12.53 \pm 1.52$ & 1.05 & 0.440 \\
\hline 5 & $16.09 \pm 2.65$ & $12.77 \pm 1.85$ & $15.17 \pm 1.67$ & $11.34 \pm 1.30$ & 0.98 & 0.311 \\
\hline 6 & $12.73 \pm 1.01$ & $10.98 \pm 1.87$ & $11.97 \pm 1.43$ & $9.66 \pm 1.57$ & 0.74 & 0.521 \\
\hline 7 & $11.04 \pm 1.34$ & $10.72 \pm 1.41$ & $10.33 \pm 1.81$ & $8.43 \pm 0.99$ & 0.69 & 0.573 \\
\hline 8 & $21.54 \pm 3.15$ & $14.18 \pm 2.13$ & $22.91 \pm 6.43$ & $20.75 \pm 9.29$ & 2.87 & 0.738 \\
\hline 9 & $18.39 \pm 4.40$ & $17.76 \pm 5.62$ & $16.39 \pm 3.18$ & $11.66 \pm 3.11$ & 2.04 & 0.671 \\
\hline 10 & $19.57 \pm 2.93$ & $16.73 \pm 2.07$ & $16.81 \pm 2.85$ & $13.67 \pm 4.08$ & 1.49 & 0.611 \\
\hline 11 & $15.84 \pm 2.55$ & $13.21 \pm 1.28$ & $13.02 \pm 1.76$ & $10.29 \pm 0.86$ & 0.90 & 0.196 \\
\hline 12 & $10.47 \pm 1.20$ & $8.07 \pm 0.48$ & $11.17 \pm 2.38$ & $9.15 \pm 0.89$ & 0.71 & 0.443 \\
\hline 13 & $11.92 \pm 2.47$ & $10.28 \pm 1.84$ & $10.27 \pm 1.87$ & $12.23 \pm 1.51$ & 0.93 & 0.835 \\
\hline 14 & $11.51 \pm 1.29$ & $9.28 \pm 0.87$ & $8.70 \pm 0.74$ & $9.64 \pm 1.43$ & 0.57 & 0.341 \\
\hline 15 & $8.96 \pm 1.01$ & $10.26 \pm 1.72$ & $7.64 \pm 0.88$ & $8.60 \pm 1.20$ & 0.61 & 0.530 \\
\hline 16 & $10.76 \pm 1.49$ & $10.72 \pm 2.02$ & $7.94 \pm 0.67$ & $11.42 \pm 2.47$ & 0.88 & 0.538 \\
\hline 17 & $11.41 \pm 1.64$ & $13.45 \pm 2.04$ & $9.99 \pm 1.12$ & $11.83 \pm 2.06$ & 0.86 & 0.588 \\
\hline 18 & $11.05 \pm 1.80$ & $9.16 \pm 1.18$ & $10.17 \pm 1.74$ & $9.21 \pm 0.80$ & 0.69 & 0.763 \\
\hline 19 & $11.64 \pm 0.66$ & $9.01 \pm 1.09$ & $9.25 \pm 0.61$ & $9.55 \pm 1.21$ & 0.49 & 0.202 \\
\hline 20 & $11.87 \pm 1.46$ & $14.35 \pm 1.63$ & $12.50 \pm 2.32$ & $14.27 \pm 2.47$ & 0.97 & 0.764 \\
\hline 21 & $12.30 \pm 2.30$ & $9.62 \pm 1.19$ & $9.95 \pm 1.28$ & $11.24 \pm 2.34$ & 0.89 & 0.726 \\
\hline 22 & $15.35 \pm 1.58$ & $12.25 \pm 2.00$ & $9.91 \pm 1.14$ & $11.14 \pm 1.58$ & 0.86 & 0.131 \\
\hline 23 & $13.42 \pm 2.82$ & $9.09 \pm 0.75$ & $11.47 \pm 1.14$ & $12.47 \pm 2.37$ & 0.97 & 0.460 \\
\hline 24 & $10.28 \pm 0.81$ & $9.07 \pm 0.83$ & $9.60 \pm 0.57$ & $9.86 \pm 1.02$ & 0.39 & 0.768 \\
\hline Mean & $13.64 \pm 1.51$ & $11.77 \pm 1.32$ & $11.99 \pm 1.45$ & $11.33 \pm 1.30$ & 0.68 & 0.672 \\
\hline
\end{tabular}

The values are Mean \pm SE of observations on five animals in each group

Plasma cortisol (ng/mL) 
The data pertaining to plasma cortisol values have been presented in table 4 . The mean cortisol $(\mathrm{ng} / \mathrm{mL})$ value were $16.96 \pm 1.05,17.07 \pm 0.68,17.97 \pm 1.54$ and $16.23 \pm 1.05$ for T1, T2, T3 and T4 group. The plasma cortisol value altered non-significantly among all the groups in each month and overall mean value.

Table 4. Effect of different levels of dietary ME and MP on plasma cortisol $(\mathrm{ng} / \mathrm{mL})$

\begin{tabular}{|lllllll|}
\hline Month & T1 & T2 & T3 & T4 & SEM & P-value \\
\hline $\mathbf{1}$ & $17.77 \pm 2.95$ & $18.24 \pm 4.23$ & $16.53 \pm 2.33$ & $11.31 \pm 1.56$ & 1.48 & 0.342 \\
\hline $\mathbf{2}$ & $24.91 \pm 2.62$ & $18.75 \pm 3.48$ & $20.15 \pm 4.81$ & $12.56 \pm 0.26$ & 1.77 & 0.092 \\
\hline $\mathbf{3}$ & $25.19 \pm 3.43$ & $14.52 \pm 2.48$ & $26.24 \pm 4.40$ & $14.98 \pm 1.27$ & 1.85 & 0.019 \\
\hline $\mathbf{4}$ & $14.65 \pm 1.65$ & $15.96 \pm 1.26$ & $14.64 \pm 1.08$ & $13.55 \pm 2.21$ & 0.77 & 0.772 \\
\hline $\mathbf{5}$ & $13.60 \pm 1.90$ & $15.73 \pm 1.45$ & $15.18 \pm 1.82$ & $13.98 \pm 0.88$ & 0.75 & 0.749 \\
\hline $\mathbf{6}$ & $11.35 \pm 1.69$ & $13.29 \pm 1.58$ & $12.36 \pm 1.01$ & $14.21 \pm 2.07$ & 0.79 & 0.643 \\
\hline $\mathbf{7}$ & $16.18 \pm 1.22$ & $16.46 \pm 2.93$ & $15.14 \pm 3.80$ & $21.37 \pm 3.64$ & 1.52 & 0.499 \\
\hline $\mathbf{8}$ & $13.41 \pm 3.87$ & $12.52 \pm 3.53$ & $17.92 \pm 3.28$ & $22.73 \pm 3.84$ & 1.90 & 0.207 \\
\hline $\mathbf{9}$ & $16.76 \pm 3.60$ & $14.81 \pm 3.33$ & $14.73 \pm 3.74$ & $15.58 \pm 1.29$ & 1.48 & 0.965 \\
\hline $\mathbf{1 0}$ & $14.19 \pm 3.22$ & $22.79 \pm 2.20$ & $15.81 \pm 2.68$ & $14.48 \pm 3.46$ & 1.55 & 0.161 \\
\hline $\mathbf{1 1}$ & $26.47 \pm 3.58$ & $15.89 \pm 4.83$ & $19.32 \pm 4.30$ & $17.86 \pm 4.01$ & 2.13 & 0.335 \\
\hline $\mathbf{1 2}$ & $21.33 \pm 3.50$ & $12.72 \pm 3.00$ & $17.57 \pm 2.25$ & $12.24 \pm 0.23$ & 1.43 & 0.065 \\
\hline Mean & $\mathbf{1 6 . 9 6} \pm \mathbf{1 . 0 5}$ & $\mathbf{1 7 . 0 7} \pm \mathbf{0 . 6 8}$ & $\mathbf{1 7 . 9 7} \pm \mathbf{1 . 5 4}$ & $\mathbf{1 6 . 2 3} \pm 1.05$ & $\mathbf{0 . 5 4}$ & $\mathbf{0 . 7 5 2}$ \\
\hline
\end{tabular}

The values are Mean \pm SE of observations on five animals in each group

\section{Blood glutathione peroxidase activity}

The antioxidant enzymes were observed on monthly basis during the growth trial to understand the effects of different levels of energy and protein on immunity parameters. The values of glutathione peroxidase ( $\mu$ mole NADPH oxidized $/ \mathrm{g} \mathrm{Hb} / \mathrm{min}$ ) were presented in table 5 . At the start of growth trial values of GPx in group T1, T2, T3 and T4 were 21.76 $\pm 3.68,19.06 \pm 2.77,18.21 \pm 2.39$ and $21.09 \pm 2.91$ respectively. The observed values of GPx did not varied significantly in any of the month or the overall mean value.

Table 5. Effect of different levels of dietary energy and protein on glutathione peroxidase ( $\mu$ mole NADPH oxidized/g Hb/min) 


\begin{tabular}{|lllllll|}
\hline Month & T1 & T2 & T3 & T4 & SEM & P-value \\
\hline $\mathbf{1}$ & $21.76 \pm 3.68$ & $19.06 \pm 2.77$ & $18.21 \pm 2.39$ & $21.09 \pm 2.91$ & 1.42 & 0.814 \\
\hline $\mathbf{2}$ & $20.40 \pm 2.84$ & $20.55 \pm 3.01$ & $21.71 \pm 1.41$ & $24.10 \pm 3.19$ & 1.30 & 0.754 \\
\hline $\mathbf{3}$ & $23.97 \pm 3.70$ & $22.08 \pm 2.01$ & $23.26 \pm 2.19$ & $22.87 \pm 0.57$ & 1.12 & 0.954 \\
\hline $\mathbf{4}$ & $26.25 \pm 5.53$ & $24.30 \pm 2.71$ & $24.98 \pm 6.90$ & $28.31 \pm 2.89$ & 2.28 & 0.939 \\
\hline $\mathbf{5}$ & $28.25 \pm 9.73$ & $26.80 \pm 4.27$ & $23.05 \pm 2.32$ & $25.56 \pm 4.12$ & 2.74 & 0.933 \\
\hline $\mathbf{6}$ & $25.18 \pm 4.66$ & $27.74 \pm 1.44$ & $23.81 \pm 2.07$ & $23.92 \pm 3.11$ & 1.47 & 0.787 \\
\hline $\mathbf{7}$ & $25.59 \pm 3.64$ & $24.65 \pm 2.65$ & $22.28 \pm 3.14$ & $25.39 \pm 3.10$ & 1.49 & 0.872 \\
\hline $\mathbf{8}$ & $23.90 \pm 2.99$ & $27.63 \pm 3.22$ & $25.93 \pm 2.60$ & $26.57 \pm 3.72$ & 1.50 & 0.863 \\
\hline $\mathbf{9}$ & $28.49 \pm 2.25$ & $26.66 \pm 1.82$ & $25.43 \pm 3.04$ & $26.39 \pm 3.76$ & 1.33 & 0.891 \\
\hline $\mathbf{1 0}$ & $29.18 \pm 5.25$ & $29.57 \pm 3.48$ & $29.82 \pm 6.47$ & $32.49 \pm 4.20$ & 2.34 & 0.964 \\
\hline $\mathbf{1 1}$ & $32.08 \pm 9.44$ & $28.30 \pm 3.66$ & $26.72 \pm 3.79$ & $24.59 \pm 1.47$ & 2.61 & 0.799 \\
\hline $\mathbf{1 2}$ & $30.68 \pm 5.44$ & $30.79 \pm 2.38$ & $26.35 \pm 1.20$ & $28.30 \pm 3.57$ & 1.68 & 0.778 \\
\hline Mean & $\mathbf{2 6 . 3 1} \pm \mathbf{2 . 9 2}$ & $\mathbf{2 5 . 6 8} \pm \mathbf{0 . 6 0}$ & $\mathbf{2 4 . 3 0 \pm 1 . 3 0}$ & $\mathbf{2 5 . 8 0} \pm 1.52$ & $\mathbf{0 . 8 5}$ & $\mathbf{0 . 8 7 4}$ \\
\hline
\end{tabular}

The values are Mean \pm SE of observations on five animals in each group

\section{Blood superoxide dismutase activity}

Monthly changes in SOD activity $(\mathrm{U} / \mathrm{mgHb})$ were recorded throughout growth trial and the values were presented in table 4.9.2. The SOD $(\mathrm{U} / \mathrm{mgHb})$ values at start of trial were $5.02 \pm 0.36,4.60 \pm 0.17,5.23 \pm$ $0.42,5.12 \pm 0.62$ in T1, T2, T3 and T4 groups respectively. The values in $3^{\text {rd }}$ month showed significant variation and the SOD $(\mathrm{U} / \mathrm{mgHb})$ values for $\mathrm{T} 1, \mathrm{~T} 2, \mathrm{~T} 3$ and $\mathrm{T} 4$ were $6.40 \pm 0.41,5.63 \pm 0.27,4.54 \pm 0.50$ and $6.00 \pm 0.23$ respectively. At ninth month T3 group $(5.04 \pm 0.37) \mathrm{SOD}(\mathrm{U} / \mathrm{mgHb})$ values was significantly lower compared to T1 group $(6.43 \pm 0.38)$. SOD also catalyses peroxide radical and functions as first intracellular defense against free radicals. The overall mean values were not significant and the values of SOD $(\mathrm{U} / \mathrm{mgHb})$ were $5.44 \pm 0.25,5.23 \pm 0.14,4.97 \pm 0.21$ and $5.27 \pm 0.15$ for $\mathrm{T} 1, \mathrm{~T} 2, \mathrm{~T} 3$ and $\mathrm{T} 4$ respectively.

Table 5. Effect of different levels of dietary ME and MP on SOD (U/mg Hb) 


\begin{tabular}{|lllllll|}
\hline Month & T1 & T2 & T3 & T4 & SEM & P-value \\
\hline $\mathbf{1}$ & $5.02 \pm 0.36$ & $4.60 \pm 0.17$ & $5.23 \pm 0.42$ & $5.12 \pm 0.62$ & 0.21 & 0.748 \\
\hline $\mathbf{2}$ & $4.53 \pm 0.25$ & $4.65 \pm 0.34$ & $4.89 \pm 0.27$ & $4.44 \pm 0.35$ & 0.15 & 0.751 \\
\hline $\mathbf{3}$ & $6.40^{\mathrm{b}} \pm 0.41$ & $5.63^{\mathrm{ab}} \pm 0.27$ & $4.54^{\mathrm{a}} \pm 0.50$ & $6.00^{\mathrm{b}} \pm 0.23$ & 0.22 & 0.012 \\
\hline $\mathbf{4}$ & $5.83 \pm 0.65$ & $5.54 \pm 0.53$ & $4.46 \pm 0.57$ & $4.78 \pm 0.56$ & 0.29 & 0.328 \\
\hline $\mathbf{5}$ & $5.65 \pm 0.30$ & $5.24 \pm 0.50$ & $4.73 \pm 0.49$ & $5.45 \pm 0.21$ & 0.20 & 0.406 \\
\hline $\mathbf{6}$ & $4.89 \pm 0.49$ & $4.82 \pm 0.18$ & $4.88 \pm 0.51$ & $5.36 \pm 0.47$ & 0.21 & 0.802 \\
\hline $\mathbf{7}$ & $5.24 \pm 0.31$ & $4.77 \pm 0.18$ & $5.38 \pm 0.44$ & $5.32 \pm 0.58$ & 0.20 & 0.704 \\
\hline $\mathbf{8}$ & $4.93 \pm 0.32$ & $4.71 \pm 0.35$ & $5.19 \pm 0.12$ & $4.86 \pm 0.27$ & 0.14 & 0.675 \\
\hline $\mathbf{9}$ & $\mathbf{6 . 4 3} \pm 0.38$ & $5.83^{\mathrm{ab}} \pm 0.21$ & $5.04^{\mathrm{a}} \pm 0.37$ & $6.09^{\mathrm{ab}} \pm 0.20$ & 0.18 & 0.027 \\
\hline $\mathbf{1 0}$ & $5.99 \pm 0.57$ & $5.71 \pm 0.45$ & $5.01 \pm 0.50$ & $5.05 \pm 0.48$ & 0.25 & 0.434 \\
\hline $\mathbf{1 1}$ & $5.16 \pm 0.54$ & $5.56 \pm 0.27$ & $4.94 \pm 0.45$ & $5.36 \pm 0.34$ & 0.20 & 0.735 \\
\hline $\mathbf{1 2}$ & $5.22 \pm 0.41$ & $5.70 \pm 0.42$ & $5.36 \pm 0.59$ & $5.41 \pm 0.52$ & 0.23 & 0.916 \\
\hline Mean & $\mathbf{5 . 4 4} \pm \mathbf{0 . 2 5}$ & $\mathbf{5 . 2 3} \pm \mathbf{0 . 1 4}$ & $\mathbf{4 . 9 7} \pm \mathbf{0 . 2 1}$ & $\mathbf{5 . 2 7} \pm \mathbf{0 . 1 5}$ & $\mathbf{0 . 1 0}$ & $\mathbf{0 . 4 1 2}$ \\
\hline
\end{tabular}

The values are Mean \pm SE of observations on five animals in each group

\section{Blood catalase activity}

The effects of different levels of dietary energy and proteins on blood catalase activity were observed and detailed results were presented in table 6 . The blood catalase value $(\mu$ moles of $\mathrm{H} 202$ consumed $/ \mathrm{min} / \mathrm{g}$ $\mathrm{Hb}$ ) at start of trial for $\mathrm{T} 1, \mathrm{~T} 2, \mathrm{~T} 3$ and $\mathrm{T} 4$ were $117.01 \pm 6.52,115.36 \pm 2.73,118.55 \pm 3.95$ and $127.48 \pm$ 5.31. At $2^{\text {nd }}$ month blood catalase values showed significant difference among the treatment groups and the catalase values ( $\mu$ moles of $\mathrm{H} 202$ consumed/min $/ \mathrm{g} \mathrm{Hb}$ ) for $\mathrm{T} 1, \mathrm{~T} 2$, T3 and T4 were $128.99 \pm 14.72$, $118.00 \pm 9.32,94.85 \pm 13.27,147.60 \pm 8.07$ respectively. At $7^{\text {th }}$ month $T 2$ group $(113.47 \pm 2.10)$ blood catalase value ( $\mu$ moles of $\mathrm{H} 202$ consumed $/ \mathrm{min} / \mathrm{g} \mathrm{Hb}$ ) was found to be significantly lower when compared to T1 group $(128.26 \pm 4.34)$ whereas at $8^{\text {th }}$ month T1 group $(138.12 \pm 10.38)$ values was found to significantly higher compared T2 and T3 groups with values $109.41 \pm 2.75$ and $107.89 \pm 9.34$ respectively. The overall mean values when compared to each other showed no significant differences with values $133.45 \pm 6.22,121.45 \pm 4.50,132.94 \pm 3.98$ and $132.74 \pm 2.42$ for $\mathrm{T} 1, \mathrm{~T} 2$, $\mathrm{T} 3$ and $\mathrm{T} 4$ group respectively.

Table 6. Effect of different levels of dietary ME and MP on catalase ( $\mu$ moles of $\mathrm{H} 202$ consumed $/ \mathrm{min} / \mathrm{g}$ $\mathrm{Hb})$ 


\begin{tabular}{|c|c|c|c|c|c|c|}
\hline Month & T1 & T2 & T3 & T4 & SEM & $\begin{array}{l}\mathrm{P} \text { - } \\
\text { value }\end{array}$ \\
\hline 1 & $117.01 \pm 6.52$ & $115.36 \pm 2.73$ & $118.55 \pm 3.95$ & $127.48 \pm 5.31$ & 2.46 & 0.318 \\
\hline 2 & $\begin{array}{l}128.99^{\mathrm{ab}} \pm \\
14.72\end{array}$ & $\begin{array}{l}118.00^{\mathrm{ab}} \pm \\
9.32\end{array}$ & $94.85^{\mathrm{a}} \pm 13.27$ & $147.60^{\mathrm{b}} \pm 8.07$ & 6.74 & 0.032 \\
\hline 3 & $145.60 \pm 10.37$ & $141.63 \pm 12.81$ & $168.53 \pm 22.26$ & $133.78 \pm 11.34$ & 7.48 & 0.415 \\
\hline 4 & $130.33 \pm 12.13$ & $121.48 \pm 6.20$ & $120.74 \pm 15.62$ & $127.17 \pm 12.04$ & 5.65 & 0.930 \\
\hline 5 & $134.62 \pm 17.05$ & $122.22 \pm 8.58$ & $137.45 \pm 17.84$ & $134.27 \pm 8.84$ & 6.55 & 0.867 \\
\hline 6 & $126.47 \pm 2.21$ & $112.90 \pm 13.07$ & $146.69 \pm 25.32$ & $\begin{array}{l}132.29 \pm \\
12.78\end{array}$ & 7.72 & 0.508 \\
\hline 7 & $128.26^{b} \pm 4.34$ & $113.47^{\mathrm{a}} \pm 2.10$ & $\begin{array}{l}118.63^{\mathrm{ab}} \pm \\
3.95\end{array}$ & $\begin{array}{l}126.11^{\mathrm{ab}} \pm \\
2.96\end{array}$ & 2.03 & 0.024 \\
\hline 8 & $138.12^{b} \pm 10.38$ & $109.41^{\mathrm{a}} \pm 2.75$ & $107.89^{a} \pm 9.34$ & $\begin{array}{l}135.56^{\mathrm{ab}} \pm \\
4.04\end{array}$ & 4.54 & 0.011 \\
\hline 9 & $140.15 \pm 7.46$ & $136.18 \pm 11.32$ & $156.28 \pm 18.05$ & $143.55 \pm 7.87$ & 5.79 & 0.667 \\
\hline 10 & $131.45 \pm 10.59$ & $126.72 \pm 5.08$ & $144.41 \pm 17.59$ & $121.99 \pm 8.35$ & 5.58 & 0.551 \\
\hline 11 & $145.93 \pm 16.05$ & $114.89 \pm 5.05$ & $141.45 \pm 16.71$ & $127.08 \pm 6.18$ & 6.26 & 0.292 \\
\hline 12 & $134.53 \pm 5.96$ & $125.15 \pm 10.70$ & $139.83 \pm 17.91$ & $136.05 \pm 12.13$ & 5.90 & 0.859 \\
\hline Mean & $133.45 \pm 6.22$ & $121.45 \pm 4.50$ & $132.94 \pm 3.98$ & $132.74 \pm 2.42$ & 2.34 & 0.205 \\
\hline
\end{tabular}

The values are Mean \pm SE of observations on five animals in each group

\section{Blood immunoglobin G}

To check immunity status of animals in the experiment trial blood IgG levels were recorded on monthly intervals and the values were presented in table 7. The values observed for $\mathrm{lgG}(\mathrm{mg} / \mathrm{mL})$ were varied not significantly among different groups. At start of trial values for T1, T2, T3 and T4 were $24.15 \pm 4.08$, $27.56 \pm 1.55,31.15 \pm 2.52$ and $28.81 \pm 2.33$ respectively. The values ranged from 24.15 to $37.96 \mathrm{lgG}$ $(\mathrm{mg} / \mathrm{mL})$. At end of growth trial values were $37.96 \pm 3.65,31.97 \pm 2.80,37.29 \pm 1.93$ and $34.66 \pm 1.33$ for $\mathrm{T} 1, \mathrm{~T} 2, \mathrm{~T} 3$ and T4 groups and the corresponding values of overall mean were $31.21 \pm 1.38,31.62 \pm 0.97$, $30.32 \pm 2.58$ and $32.25 \pm 0.64$ respectively.

Table 7. Effect of different levels of dietary ME and MP on $\operatorname{lgG}(\mathrm{mg} / \mathrm{mL})$ 


\begin{tabular}{|lllllll|}
\hline Month & T1 & T2 & T3 & T4 & SEM & P-value \\
\hline $\mathbf{1}$ & $24.15 \pm 4.08$ & $27.56 \pm 1.55$ & $31.15 \pm 2.52$ & $28.81 \pm 2.33$ & 1.40 & 0.369 \\
\hline $\mathbf{2}$ & $28.77 \pm 3.47$ & $35.00 \pm 4.04$ & $30.17 \pm 2.86$ & $33.34 \pm 3.52$ & 1.58 & 0.514 \\
\hline $\mathbf{3}$ & $31.09 \pm 2.81$ & $32.29 \pm 3.38$ & $34.25 \pm 5.38$ & $27.42 \pm 2.97$ & 1.84 & 0.636 \\
\hline $\mathbf{4}$ & $29.66 \pm 1.50$ & $30.40 \pm 1.68$ & $31.08 \pm 4.98$ & $32.98 \pm 2.21$ & 1.50 & 0.305 \\
\hline $\mathbf{5}$ & $29.64 \pm 3.78$ & $28.70 \pm 4.10$ & $35.14 \pm 2.13$ & $33.53 \pm 3.36$ & 1.69 & 0.504 \\
\hline $\mathbf{6}$ & $36.96 \pm 4.28$ & $30.60 \pm 3.45$ & $32.26 \pm 2.47$ & $32.48 \pm 3.03$ & 1.67 & 0.502 \\
\hline $\mathbf{7}$ & $25.15 \pm 3.89$ & $29.39 \pm 0.97$ & $32.29 \pm 2.76$ & $29.67 \pm 2.48$ & 1.38 & 0.346 \\
\hline $\mathbf{8}$ & $33.77 \pm 3.30$ & $36.62 \pm 3.17$ & $31.34 \pm 3.35$ & $34.51 \pm 3.14$ & 1.47 & 0.545 \\
\hline $\mathbf{9}$ & $33.09 \pm 2.80$ & $34.44 \pm 2.18$ & $30.18 \pm 3.06$ & $30.25 \pm 1.67$ & 1.22 & 0.547 \\
\hline $\mathbf{1 0}$ & $31.00 \pm 2.05$ & $31.67 \pm 2.11$ & $34.51 \pm 2.73$ & $34.32 \pm 1.92$ & 1.09 & 0.588 \\
\hline $\mathbf{1 1}$ & $33.24 \pm 2.53$ & $30.76 \pm 4.06$ & $35.14 \pm 2.13$ & $34.97 \pm 2.66$ & 1.42 & 0.700 \\
\hline $\mathbf{1 2}$ & $37.96 \pm 3.65$ & $31.97 \pm 2.80$ & $37.29 \pm 1.93$ & $34.66 \pm 1.33$ & 1.30 & 0.363 \\
\hline Mean & $31.21 \pm 1.38$ & $31.62 \pm 0.97$ & $30.32 \pm 2.58$ & $32.25 \pm 0.64$ & $\mathbf{1 . 1 6}$ & $\mathbf{0 . 2 0 5}$ \\
\hline
\end{tabular}

The values are Mean \pm SE of observations on five animals in each group

\section{Discussion}

\section{Body weight changes}

The mean body weight changes did not varied significantly in any of the fortnight or in the overall mean value. The result may be attributed to higher energy and protein provided in treatment rations is not sufficient to cause gain in body weight and this extra energy and protein is either not utilized or aiding in some other production parameters. Mahmoudzadeh et. al. (2007) fed three levels of energy and two levels of proteins in 15 months male buffalo calves and results of this study showed no final body weight difference among treatment which was in support to results of this study. Patil (2013) fed $10 \%$ higher and $20 \%$ higher energy than control (NRC, 2001) to Murrah buffalo calves and also observed no alteration in body weight changes in different groups.

\section{Dry matter intake and Feed conversation ratio}

There was no change in total dry matter intake and feed conversion ratio during the feeding trial of the study. This could be possibly due to increase in ME and MP in ration did not affect feed intake and as there was also no change in total body weight gain, therefore no alteration was observed in feed conversion ratio too. Brown et. al. (2005) analyzed DMI on heifer calves by feeding increasing levels of 
protein. Results of this study showed no significant difference in DMI $(\mathrm{kg} / \mathrm{d})$ indicating similar result to this study. Tauqir et. al. (2011) fed three levels of CP and two levels of ME, result of this study DMI had not changed significantly when CP was fed at low (11.85\%) and medium (14.25\%) level

\section{Blood antioxidant and immunity parameters}

Parameters like blood GPx, SOD, catalase and IgG were not altered during the trial indicating no type of stress and no variation in health and immunity status of animals. GPx plays key role in antioxidant defence system by decreasing $\mathrm{H}_{2} \mathrm{O}_{2}$ and other peroxides whereas SOD also catalyses peroxide radical and functions as first intracellular defense against free radicals. Values did not vary during the study indicated no harmful effects of providing higher energy and protein on health and immunity parameters.

From the present study it can be concluded that feeding 30\% higher ME and MP did not aided in body weight changes and in dry matter intake. Higher ME amd MP also did not caused any variation in stress and immunity parameters of Murrah buffalo. Therefore upto 30\% extra ME and MP could be fed in growing Murrah buffalo calves without causing any stress though it did not increased dry matter intake and body weight changes.

\section{Declarations}

\section{Acknowledgements}

Thanks to NDRI for providing necessary funds and resources for the smooth conduct of trial.

Funding: NDRI (National Dairy Research Institute)

Conflict of interest: There is no conflict of interest among authors.

Ethics approval: The experiment was approved and conducted under the established rules of the Institutional animal ethics committee (IAEC), constituted as per form no. 13 of the committee for the purpose of control and supervision of experiments on animals (CPCSEA) rules laid down by the government authority.

Consent to participate: All the authors were aware regarding participation and publications.

Consent for publication: All the authors were aware regarding participation and publications.

Availability of data and material: Data is presented from the research work conducted and also cited in the thesis.

Code availability: not applicable

Authors contribution: All the authors work in coordinated way. Experiment is planned and conducted by Prince Chauhan under the guidance of R M. S and D D helps in processing and collecting samples. Z B 
helps statistical analysis of the data. All the authors read and approved the manuscript.

\section{References}

1. BAHFS. (2015). Basic Animal Husbandry and Fishery Statistics. Government of India, Ministry of agriculture \& farmers welfare, Department of Animal Husbandry, Dairying and fisheries Krishi Bhawan, New Delhi.

2. Bhatti, S. A., Sarwar, M., Khan, M. S., \& Hussain, S. M. I. (2007). Reducing the age at first calving through nutritional manipulations in dairy buffaloes and cows: A review. Pakistan Veterinary Journal, $27(1), 42$.

3. Brown, E.G., VandeHaar, M.J., Daniels, K.M., Liesman, J.S., Chapin, L.T., Keisler, D.H., Weber Nielsen, M.S., (2005). Effect of increasing energy and protein intake on body growth and carcass composition of Heifer calves. J. Dairy Sci. 88, 585-594

4. Hosseini, S.M., Akbary, S.M., Maherisis, N. and Aghsaghali, A.M. (2008). Effect of different energy levels of diet on feed efficiency, growth rate and carcass characteristics of fattening lambs. Iranian J. Anim. and Vet. Adv., 7: 1551-1554.

5. ICAR. (2013). Nutrient composition of Indian feeds and fodders, 1st edn, ICAR and NIANP.

6. Mahmoudzadeh, H., Fazaeli, H., Kordnejad, I., \& Mirzaei, H. R. (2007). Response of male buffalo calves to different levels of energy and protein in finishing diets. Pakistan journal of biological sciences: PJBS, 10(9), 1398-1405.

7. Meacham, T.N., Cunha, T.J., Warnick, A.C., Hentges, J.F. and Hargrove, D.D. (1963). Influence of low protein rations on growth and semen characteristics of young beef bull. J. Anim. Sci., 22: 115-120.

8. NRC. (2001). Nutrient Requirements of Dairy Cattle.7th Revised Edition. National Academy Press, Washington, DC.

9. Olafadehan, O. A. and Adewumi, M. K., (2009). Productive and reproductive performance of strategically supplemented free grazing prepartum Bunaji cows in the agro pastoral farming system, $41,1275-1281$.

10. Patil, L.N. (2013).Effect of feed energy level on nutrient utilisation and growth in buffalo male calves. M.V.Sc. Thesis, NDRI, Deemed University, Karnal, Haryana, India.

11. Paul, S. S., Mandal, A. B., Kannan, A., Mandal, G. P., \& Pathak, N. N. (2003). Comparative dry matter intake and nutrient utilisation efficiency in lactating cattle and buffaloes. Journal of the Science of Food and Agriculture, 83(4), 258-267.

12. Tauqir, N. A., Shahzad, M. A., Nisa, M., Sarwar, M., Fayyaz, M., \& Tipu, M. A. (2011). Response of growing buffalo calves to various energy and protein concentrations. Livestock Science, 137(1-3), 66-72.I.

13. Vohra, V., Chopra, A. \& Raja, K.N. (2016). Diversity of buffalo genetic resources of India and its conservation. In:Conservation of indigenous domestic animal diversity, ICAR-National Beauro of Animal Genetic Resources, Karnal (India), 3, 29-43. 